\title{
Correction to: Spherical cavity-expansion model for penetration of reinforced-concrete targets
}

\author{
Y. J. Deng ${ }^{1,2,3} \cdot$ W. J. Song ${ }^{4}$ X. W. Chen ${ }^{5,6}$
}

Published online: 23 May 2019

(c) The Chinese Society of Theoretical and Applied Mechanics and Springer-Verlag GmbH Germany, part of Springer Nature 2019

\section{Correction to: \\ Acta Mechanica Sinica \\ https://doi.org/10.1007/s10409-018-0821-9}

In the original publication, Figs. 12, 13, 17 and 18 are incorrectly published. The correct versions of figures are provided in this correction.

The second sentence in the third paragraph of Sect. 5.1 "Test of Canfield and Clator" should read as "As shown in Fig. 18, the penetration depth obtained using the compressible model for reinforced concrete was slightly lower than that of Luk and Forrestal's model [25]".

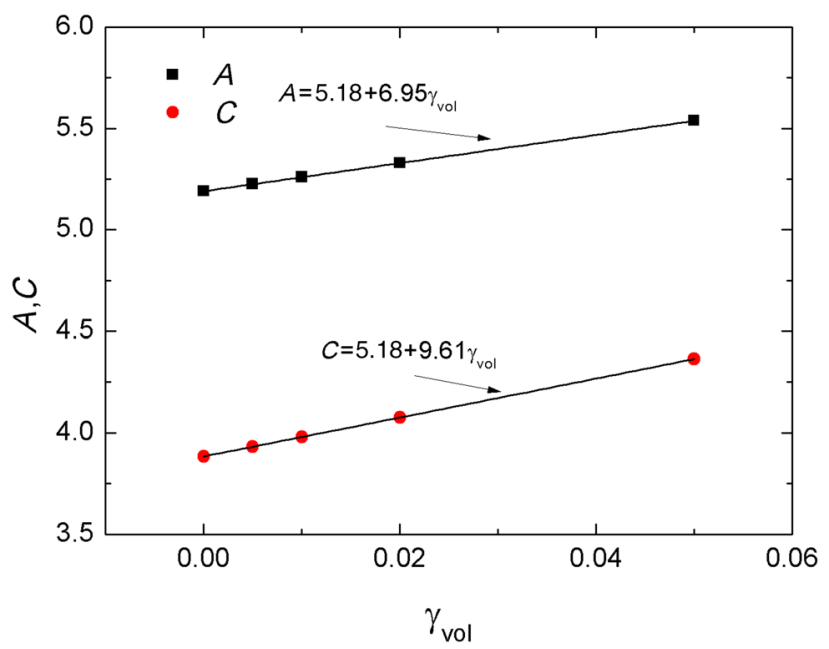

Fig. 12

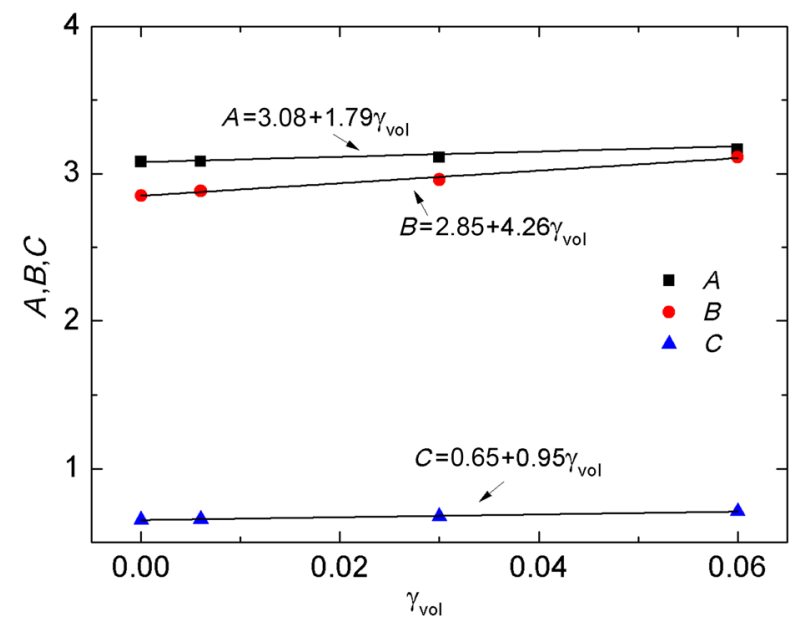

Fig. 13
The original article can be found online at https://doi.org/10.1007/ s10409-018-0821-9.

X. W. Chen

chenxiaoweintu@bit.edu.cn

1 Institute of Systems Engineering, China Academy of Engineering Physics, P.O. Box 919-416, Mianyang 621999, China

2 Shock and Vibration of Engineering Materials and Structures Key Laboratory of Sichuan Province, Mianyang 621000, China

3 Department of Civil Engineering and Architecture, Southwest University of Science and Technology, Mianyang 621000, China

4 Department of Mechanics and Engineering Science, College of Engineering, Peking University, Beijing 100871, China

5 Advanced Research Institute for Multidisciplinary Science, Beijing Institute of Technology, Beijing 100081, China

6 State Key Lab of Explosion Science and Technology, Beijing Institute of Technology, Beijing 100081, China 


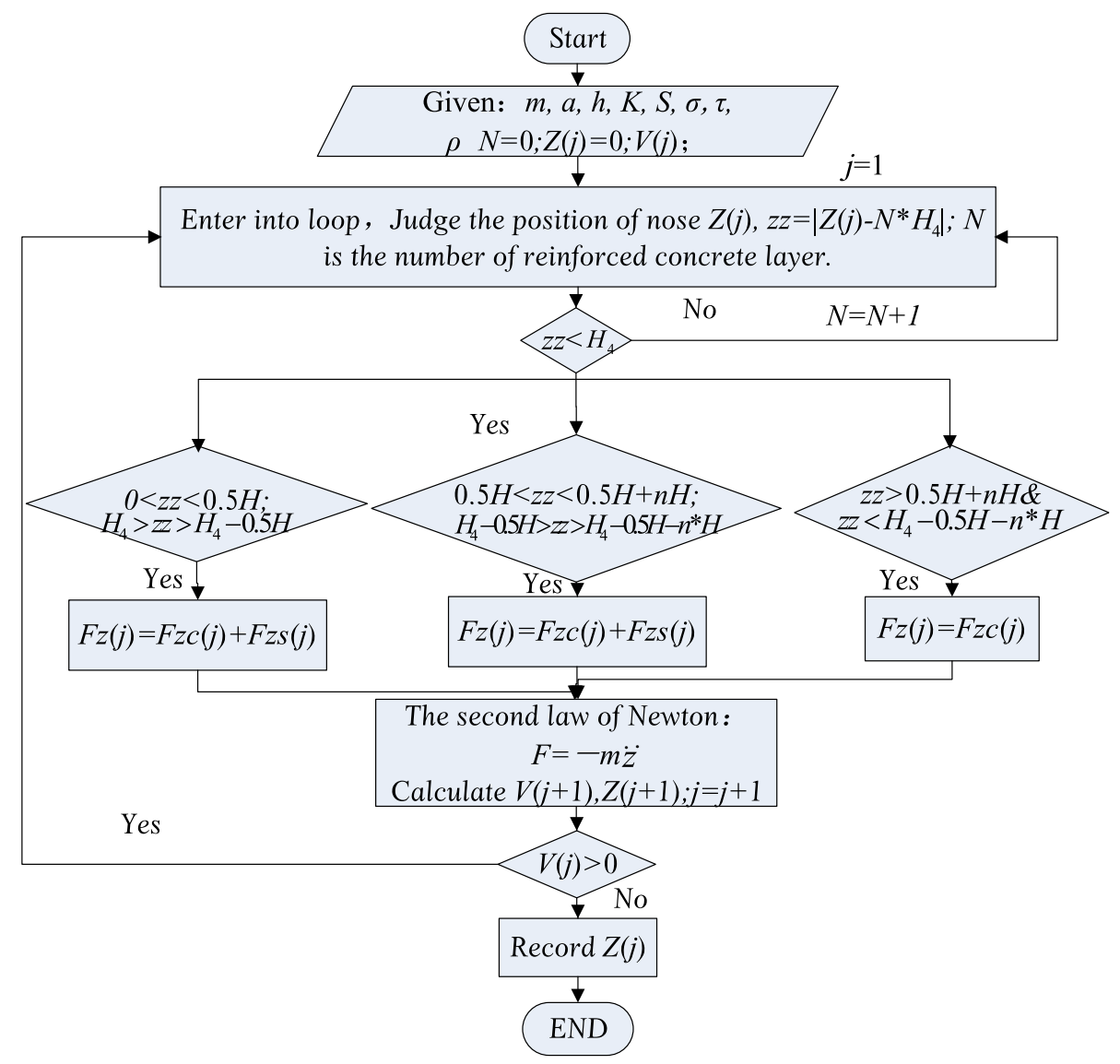

Fig. 17

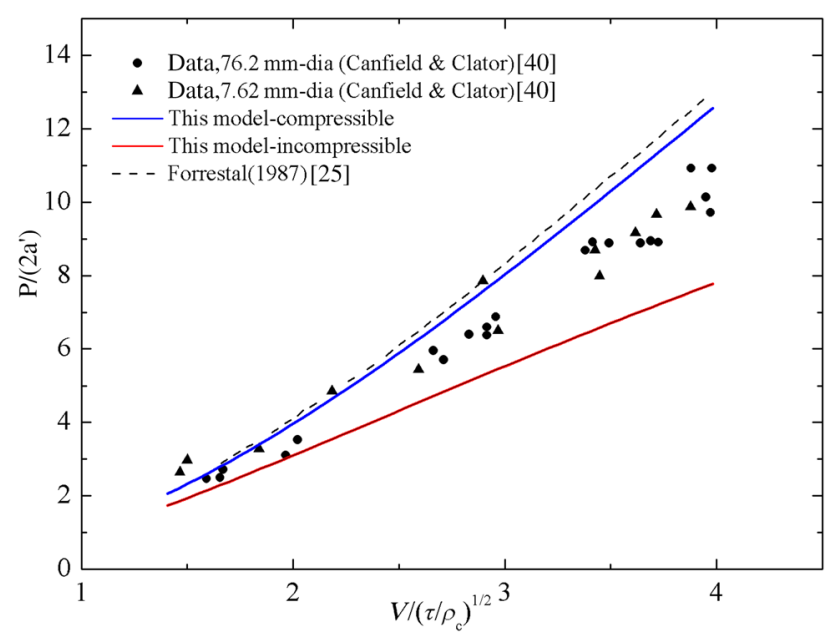

Fig. 18 